\title{
Clinical Characteristics and Risk Factors for
}

\section{Bloodstream Infection Due to Carbapenem-Resistant Klebsiella pneumoniae in Patients with Hematologic Malignancies}

This article was published in the following Dove Press journal:

Infection and Drug Resistance

\author{
Piaopiao Zhang' \\ Jie Wang ${ }^{2}$ \\ Hangbin $\mathrm{Hu} \mathbb{D D}^{\prime}$ \\ Sheng Zhang ${ }^{3}$ \\ Juying $\mathrm{Wei}^{4}$ \\ Qing Yang (D)' \\ Tingting $\mathrm{Qu}^{\prime}$
}

'State Key Laboratory for Diagnosis and Treatment of Infectious Diseases, The First Affiliated Hospital, Zhejiang University School of Medicine, Hangzhou, Zhejiang, People's Republic of China; ${ }^{2}$ Respiratory Department, The First Affiliated Hospital, Zhejiang University School of Medicine, Hangzhou, Zhejiang, People's Republic of China; ${ }^{3}$ Infection Control Department, The First Affiliated Hospital, Zhejiang University School of Medicine, Hangzhou, Zhejiang, People's Republic of China; ${ }^{4} \mathrm{Hematological}$ Diseases Department, The First Affiliated Hospital, Zhejiang University School of Medicine, Hangzhou, Zhejiang, People's Republic of China
Correspondence: Tingting Qu State Key Laboratory for Diagnosis and Treatment of Infectious Diseases, The First Affiliated Hospital, Zhejiang University School of Medicine, 79\# Qingchun East Road, Hangzhou 31000I, People's Republic of China

Tel/Fax +86 57/87236673

Email qutingting@zju.edu.cn
Purpose: The aim was to examine the clinical characteristics and risk factors for bloodstream infection (BSI) due to carbapenem-resistant Klebsiella pneumoniae (CRKP) in patients with hematologic malignancies.

Materials and Methods: A single-centre, retrospective case-control study representing 734 patients with hematologic malignancies between January 1, 2017, and December 31, 2018, was conducted. Demographic and clinical data were collected from the hospital electronic medical records system.

Results: Among the 734 patients with hematologic malignancies, 3\% (22/734) of the patients developed CRKP BSI during their hospitalization. Overall 28-day all-cause mortality reached $77.3 \%$ (17/22). Patients with Pitt bacteremia score (PBS) $>4$, pneumonia and septic shock were more frequent in the non-survivors versus the survivors. Compared with the non-survivors in antimicrobial treatment, combination therapy of tigecycline and polymyxin B was more common in the survivors. The independent risk factors associated with CRKP BSI were CRKP rectal colonization (OR, 11.067; CI=4.43-27.644; $\mathrm{P}<0.001 ; 3$ points), severe neutropenia ( $\mathrm{OR}, 4.095 ; \mathrm{CI}=0.876-19.141 ; \mathrm{P}=0.073 ; 1$ point) and invasive mechanical ventilation (IMV) within the previous 30 days to onset of BSI (OR, 18.444; $\mathrm{CI}=1.787-190.343 ; \mathrm{P}=0.014 ; 4$ points). The total risk score of $\geq 5$ indicated that the probability of CRKP BSI occurrence was above $48 \%$.

Conclusion: CRKP BSI in patients with hematologic malignancies is associated with high mortality. The risk factor-based prediction model might help clinicians to start prompt effective anti-infective therapy in patients with suspicion of CRKP BSI and improve outcomes.

Keywords: carbapenem-resistant Klebsiella pneumoniae, bloodstream infection, hematologic malignancy, risk factors, prediction

\section{Introduction}

Carbapenem-Resistant Enterobacteriaceae (CRE) has emerged as a significant public health threat worldwide, with very few available effective treatment options. ${ }^{1,2}$ Over the past decade in China, the annual isolation rate of carbapenemresistant Klebsiella pneumoniae (CRKP) steadily increased, from 3.0\% in 2005 to $20.9 \%$ in 2017, ranking first among the isolated CRE, followed by E. coli and Enterobacter cloacae. ${ }^{3-5}$ Immunocompromised hosts, especially patients with 
hematologic malignancies, are at increased risk for CRKP infections due to the chemotherapy-induced gastrointestinal mucositis, myelosuppression after chemotherapy, prolonged hospital stays and frequent exposure to broadspectrum antimicrobial therapy. ${ }^{6-8}$

CRKP bloodstream infections (BSIs) are associated with higher mortality than other infection types of CRKP and require special treatments timely, especially in hematological patients. In the United States, up to half of all BSIs caused by CRE resulted in death each year, of which carbapenem-resistant Klebsiella spp. and carbapenemresistant $E$. coli were the most common two pathogens. ${ }^{9}$ Previous researches have shown that CRKP BSIassociated fatality rate occurred based on hematologic malignancies exceeds $50 \%{ }^{10-13}$

To improve the efficacy of empirical therapy and reduce CRKP BSI-related mortality in patients with hematologic malignancies, this study was conducted to establish a risk prediction model of CRKP BSI, and seek appropriate treatment in this population.

\section{Materials and Methods}

\section{Ethics}

All isolates present in this study were stored in the Department of Microbiology, the First Affiliated Hospital, Zhejiang University School of Medicine. The study was approved by the ethical research committee of the First Affiliated Hospital, College of Medicine, Zhejiang University. The ethics committee approved the waiver of patient's informed consent, with the justification that this was a retrospective and analytical study whose information was obtained from medical records and that the data were de-identified and anonymously analyzed. The study was performed in accordance with the Declaration of Helsinki and all its following amendments. Privacy statement: the authors guarantee patient data confidentiality.

\section{Study Design and Participants}

A single-centre, retrospective, case-control study of patients with hematologic malignancies who were hospitalized between January 1st, 2017, and December 31th, 2018, was performed at The First Affiliated Hospital of Zhejiang University in Hangzhou, China. Patients admitted for less than seven days were excluded. Episodes of CRKP BSI were identified based on blood culture results. All the cases with CRKP BSI were followed for 28 days and were divided into survival and non-survival group. Clinical characteristics and risk factors of CRKP BSI were explored (Figure 1).

\section{Data Collection}

Demographic and clinical data were extracted from the hospital electronic medical records system. Clinical data of the patients with BSI were collected regarding gender, age, diagnosis, severe neutropenia, complications (pneumonia, septic shock), PBS, appropriate initial antiinfection therapy, and anti-CRKP therapeutic regimen. The risk factor variables were as follows: age; gender; diagnosis; leukemia induction; hematopoietic stem-cell transplantation (HSCT); graft versus host disease (GVHD); CRKP rectal colonization; severe neutropenia; gastrointestinal disturbance; renal insufficiency; antibiotic exposure; the history of intensive care unit (ICU), invasive mechanical ventilation (IMV), urinary catheter and peripherally inserted central catheter (PICC) or central venous catheter (CVC) within the 30 days before the onset of CRKP BSI.

\section{Definitions}

CRKP BSI was defined as the positive blood culture for $K$. pneumoniae with resistance to any carbapenem (intermediately susceptible results were reported as resistant). ${ }^{9}$ The date when the first positive blood culture was drawn was considered as the onset of BSI. The presence of CRKP in rectal swab screenings during hospitalization was deemed to be CRKP rectal colonized. Severe neutropenia was defined as an absolute neutrophil count $<100$. Having one of the following conditions was defined as gastrointestinal disturbance: intestinal graft-versus-host disease, Clostridium difficile infection, diarrhea lasted more than three days. Renal insufficiency was defined as a creatinine clearance of $<30$. Antibiotic exposure meant that antibiotics were administered intravenously or orally more than 48 hours within 90 days before BSI or discharge. Anti-anaerobic agents referred to metronidazole and piperacillin-tazobactam. ${ }^{14}$ PBS was calculated within 48 hours before or on the day of first positive blood culture, and the highest point score during that time was recorded. Appropriate initial anti-infection therapy was defined as antibiotics administered within 48 hours of BSI that appeared to be active against CRKP when antimicrobial susceptibility test results were available. All-cause mortality was defined as death from any cause during hospitalization. 


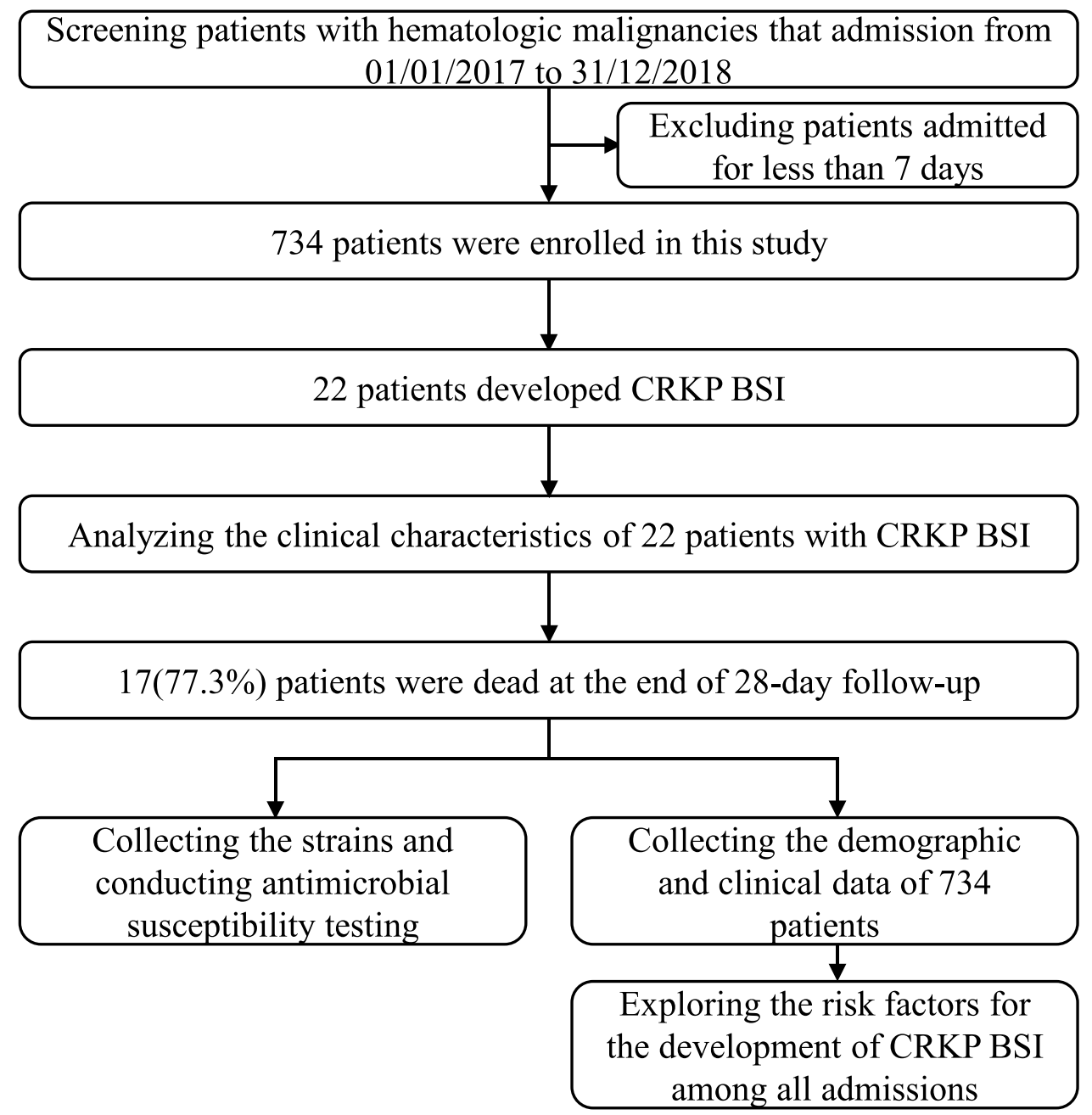

Figure I Flowchart of the study design.

\section{Statistical Analysis}

Median and interquartile range (IQR) were used to describe the non-normal distribution of continuous variables. Number and percentage were reported for categorical variables. In the univariate analysis, categorical variables were compared using $\chi^{2}$ or Fisher's exact test while continuous variables were analyzed using the Mann-Whitney $U$-test for nonparametrically distributed data. The strength of the correlation was expressed as the odds ratio (OR) and 95\% confidence intervals (95\% CI). All analyses were 2-tailed. A P value $<0.05$ was considered statistically significant. Candidate variables with $\mathrm{p}<0.2$ or clinically regarded as significant in the univariate analysis were subsequently included in the multivariable analysis, from which we obtained independent risk factors for CRKP BSI, $\mathrm{P}$ value $<0.1$ being statistically significant. The regression coefficients of each independent risk factor were simultaneously divided by the minimum one, taking the result as the risk score of the corresponding variable. A risk prediction model was established based on the binary logistic regression equation. The prediction performance and fitting degree of the model were evaluated by the receiver operating characteristic (ROC) curve and Hosmer-Lemeshow test, respectively. A 28-day univariate survival analysis of patients with CRKP BSI was performed by the Kaplan-Meier method. Data were processed in SPSS version 25.0.

\section{Collection and Identification of Isolates}

Twenty-two CRKP strains were isolated from patients' blood who developed CRKP BSI. After obtaining single colonies, all isolates were identified and reidentified using an automated Vitek 2 system (bioMérieux, France). 


\section{Antibiotic Susceptibility Testing}

We conducted antibiotic susceptibility testing on the 22 CRKP strains using agar dilution and broth dilution methods. Antimicrobial agents tested included amikacin, aztreonam, ceftriaxone, cefoxitin, levofloxacin, sulfamethoxazole, cefepime, piperacillin-tazobactam, cefoperazone-sulbactam, tigecycline, polymyxin B. The tigecycline and polymyxin $B$ results were interpreted according to the criteria and recommendations from European Committee on Antimicrobial Susceptibility Testing (EUCAST, 2019) while the remaining antimicrobial results were determined in accordance with Clinical and Laboratory Standards Institute (CLSI, 2019). E. coli ATCC 25922 was used as a control strain for the susceptibility test.

\section{Results}

\section{Clinical Characteristics of Patients with CRKP BSI}

A total of 734 patients with hematologic malignancies were included in this study, of which 22 (3\%) developed CRKP BSI. The 28-day all-cause fatality rate was up to $77.3 \%(17 / 22)$.

The number of male patients was more than twice as likely as female patients. AML accounted for the largest proportion $(63.6 \%)$ of the underlying diseases. Eighteen (81.8\%) patients had severe neutropenia after BSI. Pneumonia and septic shock were both observed in 13 (59.1\%) patients. Sixteen $(72.7 \%)$ patients accepted the appropriate initial anti-infection therapy (Table 1).

The median age of the non-survival group was higher than that of the survival group. PBS $>4$, pneumonia and septic shock were more common in the non-survival group ( $17.6 \%$ vs $0 \% ; 64.7 \%$ vs $40 \%, 76.5 \%$ vs $0 \%$ ). All of the survivors received appropriate initial anti-infection therapy, but only $11(64.7 \%)$ in the non-survivors. Two $(40 \%)$ patients in the survival group were treated with tigecycline combined with polymyxin $\mathrm{B}$, while just 5 $(29.4 \%)$ in the non-survival group (Table 2).

Kaplan-Meier curve revealed that $\mathrm{PBS}<4 \quad(\mathrm{P}<0.001)$ (Figure 2A) and tigecycline combined with polymyxin $\mathrm{B}$ as anti-CRKP therapeutic regimen $(\mathrm{P}=0.019)$ (Figure 2B) could reduce the all-cause mortality.

\section{Risk Factors for Acquisition of CRKP BSI} Univariate analysis (Table 3) revealed that patients with CRKP rectal colonization were prone to BSI $(\mathrm{P}<0.001)$, the difference being statistically significant. Multivariate logistic
Table I Clinical Characteristics of 22 Patients with CRKP BSI

\begin{tabular}{|l|l|l|}
\hline & N or Median & $\%$ \\
\hline Number of patients & 22 & \\
\hline Gender & & \\
Male & 15 & 68.2 \\
Female & 7 & 31.8 \\
Average age & 45 & \\
\hline Diagnosis & & \\
AML & 14 & 63.6 \\
ALL & 5 & 22.7 \\
MDS & 1 & 4.5 \\
Multiple Myeloma & 1 & 4.5 \\
Others & 1 & 4.5 \\
Severe neutropenia after BSI & 18 & 81.8 \\
PBS>4 & 3 & 13.6 \\
Pneumonia & 13 & 59.1 \\
Septic shock & 13 & 59.1 \\
Appropriate initial anti-infection therapy & 16 & 72.7 \\
\hline Anti-CRKP therapeutic regimen & & \\
Tigecycline & 12 & 54.5 \\
Polymyxin B & 0 & 0 \\
Tigecycline + Polymyxin B & 7 & 31.8 \\
None of the above & 3 & 13.6 \\
\hline
\end{tabular}

regression analysis identified the followings as independent risk factors for CRKP BSI: CRKP rectal colonization (OR, 11.067; $\mathrm{CI}=4.43-27.644 ; \mathrm{P}<0.001$ ), severe neutropenia (OR, 4.095; $\mathrm{CI}=0.876-19.141 ; \mathrm{P}=0.073), \quad \mathrm{IMV}(\mathrm{OR}, 18.444$; $\mathrm{CI}=1.787-190.343 ; \mathrm{P}=0.014)$.

\section{The Risk Score and Prediction Probability for Development of CRKP BSI}

The total risk score was 8 points, among which IMV was the highest (4 points), CRKP rectal colonization was 3 points and severe neutropenia was one point (Table 4). The prediction probability of CRKP BSI was $0.8 \%, 2 \%$, $12.2 \%, 26.4 \%, 48 \%, 86 \%$ and $94 \%$ with total risk score of $0,1,3,4,5,7$ and 8 , respectively. A higher score indicated a higher risk of CRKP BSI. The Hosmer- Lemeshow test demonstrated that this model had a good fit to the data $(\mathrm{P}=$ 0.236). ROC curve showed that the risk score was a good predictive factor for BSI, with an AUROC of 0.753 (95\% $\mathrm{CI}=0.648-0.858$ ). (Figure 3 )

\section{Antimicrobial Agent Susceptibility Results} The antimicrobial agent susceptibilities of 22 CRKP isolates showed that $100 \%$ of the isolates were resistant to 
Table 2 Comparison of Clinical Characteristics Between the Survival Group and the Non-Survival Group

\begin{tabular}{|c|c|c|c|c|}
\hline & \multicolumn{2}{|c|}{ Survivors } & \multicolumn{2}{|c|}{ Non-Survivors } \\
\hline & $\begin{array}{l}\mathbf{N} \text { or } \\
\text { Median }\end{array}$ & $\begin{array}{l}\% \text { or } \\
\text { (IQI) }\end{array}$ & $\begin{array}{l}\mathbf{N} \text { or } \\
\text { Median }\end{array}$ & $\begin{array}{l}\text { \% or } \\
\text { (IQI) }\end{array}$ \\
\hline Number of patients & 5 & 22.7 & 17 & 77.3 \\
\hline \multicolumn{5}{|l|}{ Gender } \\
\hline Male & 4 & 80 & 11 & 64.7 \\
\hline Female & 1 & 20 & 6 & 35.3 \\
\hline Age & 38 & $26-50$ & 47 & $33-60$ \\
\hline \multicolumn{5}{|l|}{ Diagnosis } \\
\hline AML & 4 & 80 & 10 & 58.8 \\
\hline ALL & 1 & 20 & 4 & 23.5 \\
\hline MDS & 0 & 0 & 1 & 5.9 \\
\hline Multiple Myeloma & 0 & 0 & 1 & 5.9 \\
\hline Others & 0 & 0 & 1 & 5.9 \\
\hline $\begin{array}{l}\text { Severe neutropenia } \\
\text { after BSI }\end{array}$ & 4 & 80 & 14 & 82.4 \\
\hline $\mathrm{PBS}>4$ & 0 & 0 & 3 & 17.6 \\
\hline Pneumonia & 2 & 40 & 11 & 64.7 \\
\hline Septic shock & 0 & 0 & 13 & 76.5 \\
\hline $\begin{array}{l}\text { Appropriate initial anti- } \\
\text { infection therapy }\end{array}$ & 5 & 100 & 11 & 64.7 \\
\hline \multicolumn{5}{|l|}{$\begin{array}{l}\text { Anti-CRKP therapeutic } \\
\text { regimen }\end{array}$} \\
\hline Tigecycline & 3 & 60 & 9 & 52.9 \\
\hline Polymyxin B & 0 & 0 & 0 & 0 \\
\hline Tigecycline+ Polymyxin B & 2 & 40 & 5 & 29.4 \\
\hline None of the above & 0 & 0 & 3 & 17.7 \\
\hline
\end{tabular}

ceftriaxone, cefoxitin, cefepime, piperacillin-tazobactam, cefoperazone-sulbactam; $95.5 \%$ resistant to levofloxacin; $89.4 \%$ resistant to aztreonam; $81.8 \%$ resistant to amikacin; $72.7 \%$ resistant to sulfamethoxazole. Only one isolate was detected resistant to tigecycline and no isolate was resistant to polymyxin B (Figure 4). In Figure 5, antibiotic resistance rates of CRKP were compared among the survival group and the non-survival group: levofloxacin ( $87.5 \%$ vs $100 \%)$, aztreonam ( $83.3 \%$ vs $92.3 \%$ ), amikacin ( $75 \%$ vs $85.7 \%)$, sulfamethoxazole $(62.5 \%$ vs $78.6 \%)$, tigecycline ( $0 \%$ vs $11.1 \%)$.

\section{Discussion}

Carbapenem-resistant $K$. pneumoniae is an increasingly prevalent pathogen that poses a serious threat to immunocompromised hosts, particularly patients with hematologic malignancies. $^{6,11,15,16}$ Novel resistance classifications for Gram-negative bacteria suggested that an extended-spectrum (a)

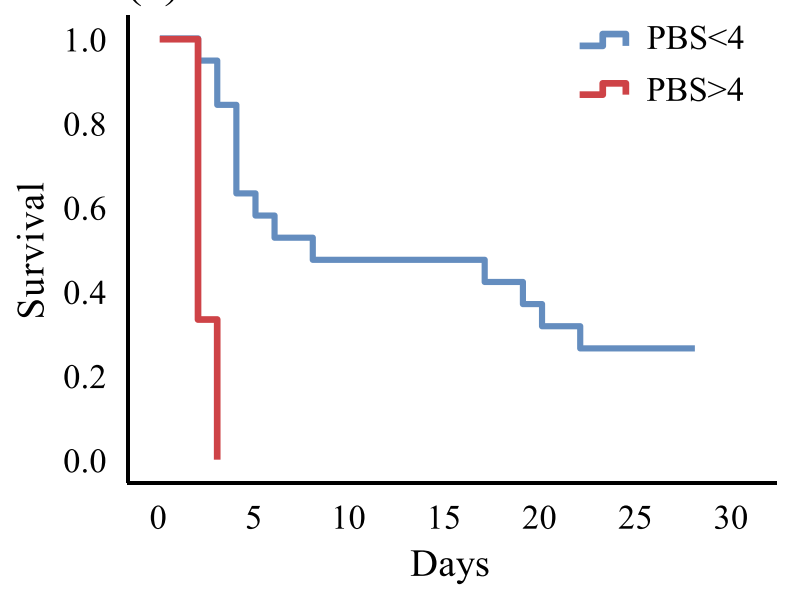

(b)

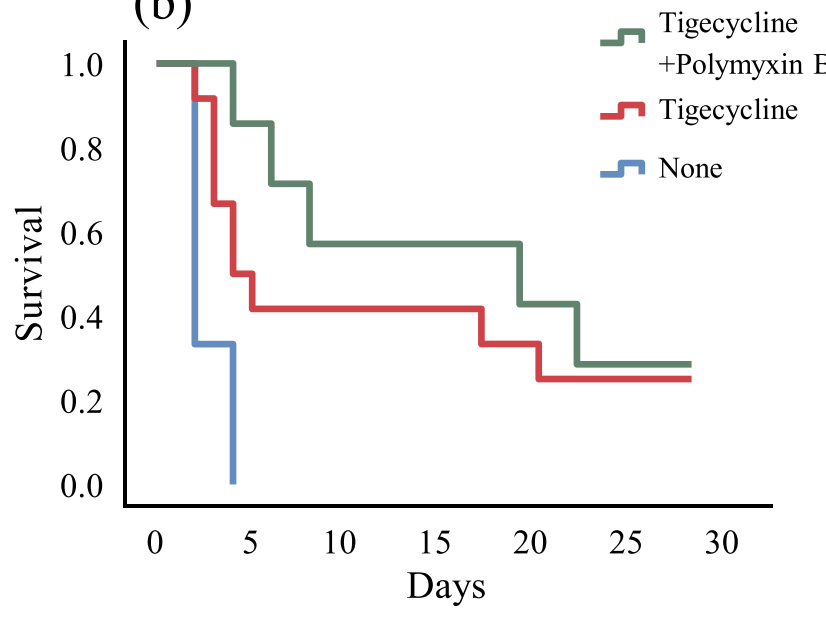

Figure 2 Kaplan-Meier univariate survival estimates (A) PBS within 48 hours before or on the day of first positive blood culture; (B) anti-CRKP therapeutic regimen after the bloodstream infection.

$\beta$-lactamase (ESBL)-producing, carbapenem-resistant and fluoroquinolone-resistant isolate was considered as difficultto-treat resistance (DTR).${ }^{17}$ Infections, especially bloodstream infections caused by CRKP can cause significantly high mortality, for the reason that it expresses multidrug resistance phenotypes so that antimicrobial treatments appear to be quite intractable. In our study, the 28-day mortality of CRKP BSI among patients with hematologic malignancies reached $77.3 \%$. It is higher compared to that reported in previous works, in which mortality rates ranged from $50 \%$ to $60 \% .^{10-}$ ${ }^{13}$ Accurate detection of high-risk CRKP BSI patients and timely implementation of infection control to contain them play a crucial role in better survival.

As is evident from Table 2, the median age of the nonsurvival group was older than that of the survival group. PBS $>4$, pneumonia and septic shock were more common in the non-survival group. All of the survivors received 
Table 3 Comparison of Patients with and without CRKP Bloodstream Infection

\begin{tabular}{|c|c|c|c|c|c|}
\hline & \multicolumn{2}{|l|}{ CRKP BSI } & \multicolumn{2}{|l|}{ No CRKP BSI } & \multirow[t]{2}{*}{$P$} \\
\hline & $\mathbf{N}$ or Median & \% or (IQI) & $\mathbf{N}$ or Median & $\%$ or (IQI) & \\
\hline Number of patients & 22 & 3 & 712 & 97 & \\
\hline Male & 15 & 62.8 & 411 & 57.7 & 0.33 \\
\hline Age & 45 & $33-59$ & 44 & $30-56$ & 0.73 \\
\hline \multicolumn{6}{|l|}{ Diagnosis } \\
\hline AML & 14 & 63.6 & 351 & 49.3 & 0.19 \\
\hline ALL & 5 & 22.7 & 152 & 21.3 & 1.00 \\
\hline MDS & 1 & 4.5 & 50 & 7 & 0.98 \\
\hline CLL/SLL & 0 & 0 & 2 & 0.3 & 1.00 \\
\hline CML & 0 & 0 & 2 & 0.3 & 1.00 \\
\hline CMML & 0 & 0 & 5 & 0.7 & 1.00 \\
\hline Multiple Myeloma & 1 & 4.5 & 50 & 7 & 0.98 \\
\hline Hodgkin Disease & 0 & 0 & 4 & 0.5 & 1.00 \\
\hline $\mathrm{NHL}$ & 0 & 0 & 87 & 12.2 & 0.16 \\
\hline Others & 1 & 4.5 & 9 & 1.2 & 0.26 \\
\hline Leukemia Induction & 5 & 22.7 & 136 & 19.1 & 0.88 \\
\hline HSCT & 7 & 31.8 & 342 & 48 & 0.13 \\
\hline Autologous HSCT & 0 & 0 & 56 & 7.9 & 0.52 \\
\hline Allogeneic HSCT & 7 & 31.8 & 286 & 40.2 & 0.43 \\
\hline GVHD & 3 & 13.6 & 102 & 14.3 & 1.00 \\
\hline CRKP Rectal Colonization & 9 & 40.9 & 45 & 6.3 & $<0.001$ \\
\hline Severe Neutropenia $(A N C<100)$ & 18 & 81.8 & 539 & 75.7 & 0.51 \\
\hline Days With ANC $<100$ & 7 & $4-11$ & 7 & $2-12$ & 0.86 \\
\hline GI Disturbance & 7 & 31.8 & 293 & 41.2 & 0.38 \\
\hline Renal Insufficiency & I & 4.5 & 15 & 2.1 & 0.39 \\
\hline Anti-anaerobic Antibiotic & 11 & 50 & 368 & 51.7 & 0.88 \\
\hline Antibiotic Days of Therapy & 2 & $0-12$ & 3 & $0-11$ & 0.55 \\
\hline 3rd-4th-Generation Cephalosporin & 9 & 40.9 & 423 & 59.4 & 0.08 \\
\hline Antibiotic Days of Therapy & 0 & $0-10$ & 5 & $0-11$ & 0.37 \\
\hline Fluoroquinolone & 10 & 45.5 & 399 & 56 & 0.33 \\
\hline Antibiotic Days of Therapy & 0 & $0-8$ & 6 & $0-10$ & 0.16 \\
\hline Aminoglycoside & 1 & 4.5 & 60 & 8.4 & 0.80 \\
\hline Antibiotic Days of Therapy & 0 & $0-0$ & 0 & $0-0$ & 0.50 \\
\hline Glycopeptide & 9 & 40.9 & 350 & 49.2 & 0.45 \\
\hline Antibiotic Days of Therapy & 0 & $0-6$ & 0 & $0-8$ & 0.28 \\
\hline Carbapenem & 16 & 72.7 & 595 & 83.6 & 0.18 \\
\hline Antibiotic Days of Therapy & 8 & $0-16$ & 10 & $5-16$ & 0.41 \\
\hline \multicolumn{6}{|l|}{ Anti-CRKP Antibiotics } \\
\hline Tigecycline & 5 & 22.7 & 175 & 24.6 & 0.84 \\
\hline Antibiotic Days of Therapy & 0 & $0-2$ & 0 & $0-0$ & 0.86 \\
\hline Polymyxin B & 1 & 4.5 & 12 & 1.7 & 0.33 \\
\hline Antibiotic Days of Therapy & 0 & $0-0$ & 0 & $0-0$ & 0.31 \\
\hline
\end{tabular}

(Continued) 
Table 3 (Continued).

\begin{tabular}{|l|l|l|l|l|l|}
\hline \multicolumn{2}{|l|}{} & \multicolumn{2}{l|}{ CRKP BSI } & \multicolumn{2}{l|}{ No CRKP BSI } \\
\cline { 2 - 6 } & N or Median & \% or (IQI) & N or Median & $\%$ or (IQI) \\
\hline ICU (within 30 days) & 1 & 4.5 & 4 & 0.6 & 0.14 \\
IMV (within 30 days) & 1 & 4.5 & 3 & 0.4 & 0.12 \\
Urinary Catheter (within 30 days) & 2 & 9.1 & 21 & 3 & 0.15 \\
PICC or CVC (within 30 days) & 22 & 100 & 657 & 92.3 & 0.35 \\
\hline
\end{tabular}

Table 4 Logistic Regression Analysis of Risk Factors for CRKP BSI Development

\begin{tabular}{|l|l|l|l|}
\hline & OR (95\% CI) & $\begin{array}{l}\boldsymbol{\beta}- \\
\text { Value }\end{array}$ & $\begin{array}{l}\text { Risk } \\
\text { Score } \\
\text { Point }\end{array}$ \\
\hline $\begin{array}{l}\text { CRKP rectal } \\
\text { colonization } \\
\text { Severe neutropenia } \\
\text { IMV }\end{array}$ & $\begin{array}{l}\text { II.067 (4.43-27.644) } \\
\text { I8.444 (1.787-190.343) }\end{array}$ & 2.519 & 3 \\
\hline
\end{tabular}

appropriate initial anti-infection therapy, while only 11 (64.7\%) patients in the non-survival group. A further study of the above 11 patients found that all had experienced severe neutropenia after the occurrence of BSI. Based on this information, we speculated that advanced age, $\mathrm{PBS}>4$, severe neutropenia, pneumonia and septic shock were death-associated risk factors, while appropriate initial anti-infection therapy is protective against mortality. Our results have been proved by Trecarichi et al. ${ }^{12,13,18-23}$ In this study, these CRKP isolates from the patients with

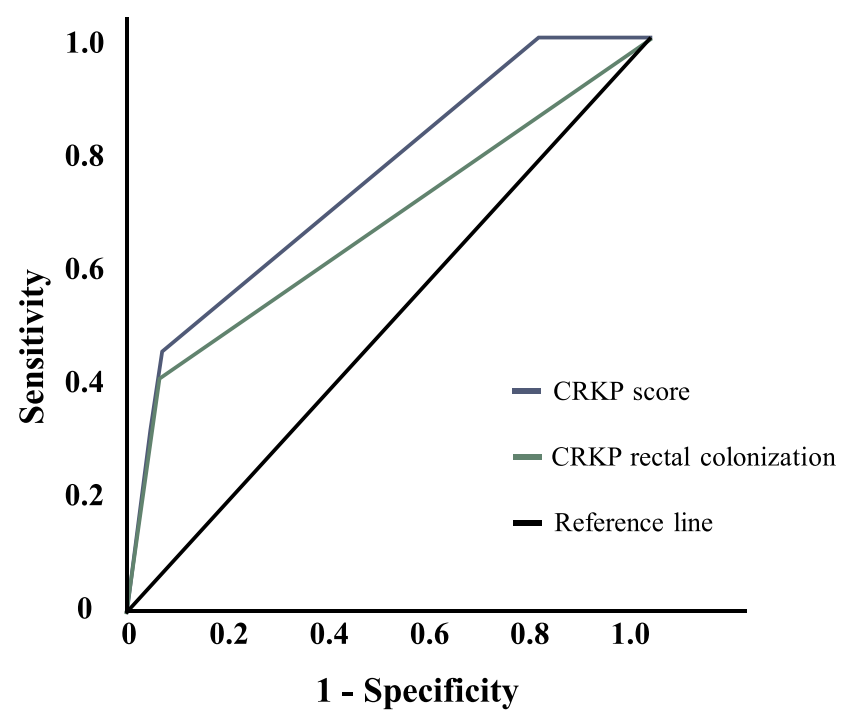

Figure 3 Receiver-operator characteristic curve for CRKP score and CRKP BSI. hematologic malignancies showed high resistance to most commonly used antimicrobial agents except tigecycline and polymyxin $\mathrm{B}$, which was in accordance with the results of the previous reports. ${ }^{4}$ Figure 5 shows that the antibiotic resistance rates to several agents (fluoroquinolones, monocyclic amides, aminoglycosides, sulfonamides, tigecycline) of the non-survival group were all significantly higher than that of the survival group, which may directly lead to the limited option of active antibiotics for patients infected with such pathogens. Figure $2 \mathrm{~B}$ reveals that tigecycline combined with polymyxin B could improve survival. The optimal treatment for invasive infections caused by CRKP has not yet been established. ${ }^{13}$ But based on observational studies, combination therapy that includes $>1$ drug with vitro activity against the isolates is significantly associated with survival. ${ }^{13,23-25}$ Mario Tumbarello reinforced concerns that monotherapy regimens utilizing drugs with substantial potency or pharmacokinetic shortcomings for BSIs may be related to increased mortality. ${ }^{23}$

When is appropriate time to empirically combat CRKP in patients undergoing hematologic malignancies remains an

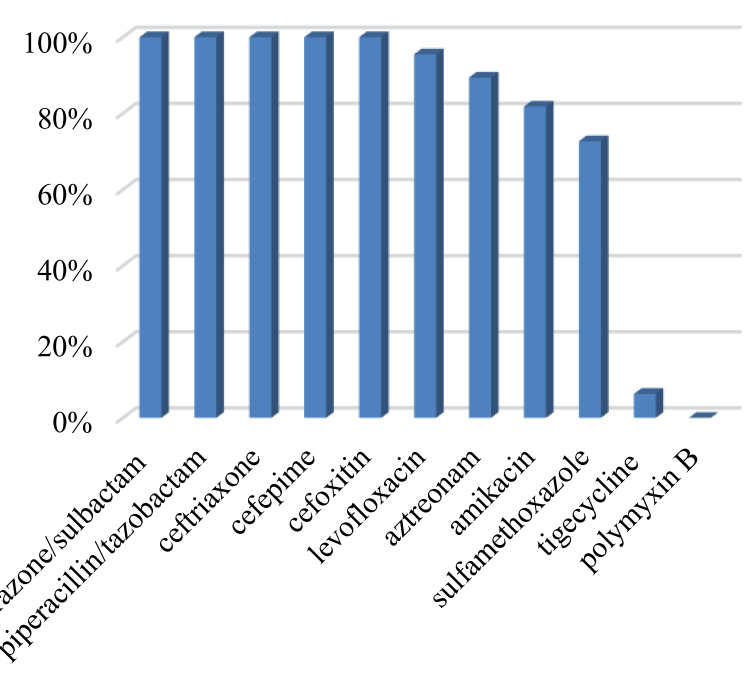

Figure 4 Antibiotic resistance rates of 22 CRKP strains. 


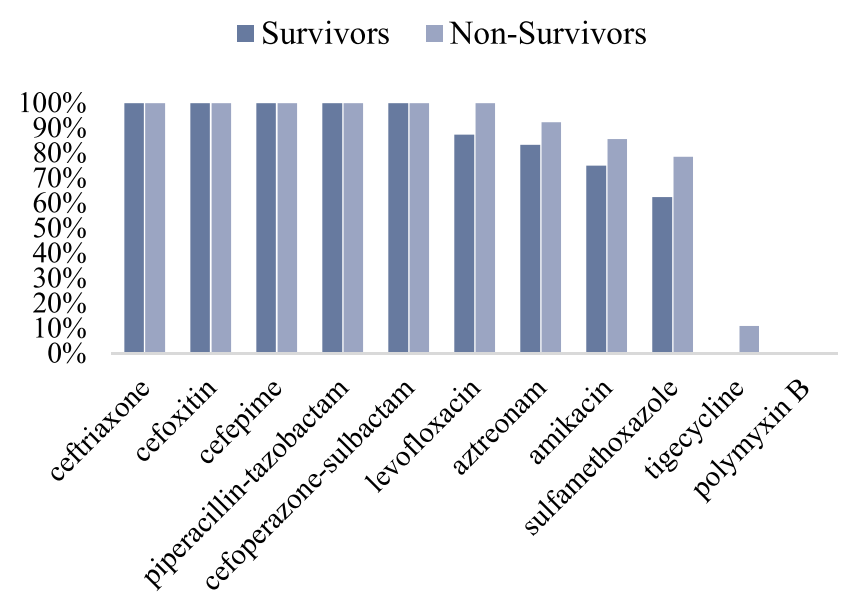

Figure 5 Comparison of antibiotic resistance rates of CRKP between the survival group and the non-survival group.

unavoidable difficult problem. Given the limited therapeutic options, prevention of CRKP infection in this vulnerable population is of paramount importance. Recently, several studies concerning risk factors for CRKP BSI have reported mostly concerning CRKP carriers and patients with immunosuppression or the ICU. There also have been similar studies in patients with hematologic malignancies, but the number of participants included is relatively small and there has no risk prediction score for it yet. The findings of our study showed that CRKP rectal colonization, severe neutropenia and IMV were independent risk factors for CRKP BSI in patients with hematologic malignancies. In accordance with the present results, the previous retrospective clinical studies have demonstrated that CRKP rectal colonization and mechanical ventilation are vital prediction factors for CRKP BSI. ${ }^{26,27}$ Malignancy hematologic patients whose immune defence function is impaired due to use systemic immunosuppressants, ${ }^{28}$ intestinal microecology is out of balance caused by exposure to a wide range of antibiotics and intestinal mucosa is damaged by chemotherapy or acute GVHD, ${ }^{29,30}$ are susceptible to develop BSI because these factors make pathogenic bacteria implanted in intestine easy to break through mucosa barrier into blood escaping from effective containment and removal. The CRKP rectal colonization rate was $7.4 \%$ in our study, significantly higher than that reported by Cattaneo et al, ${ }^{11,31}$ confirming that this is a growing problem in endemic areas of our country and great attention must be paid to it. IMV makes it vulnerable to the occurrence of ventilator-associated pneumonia, coupled with the breakdown of the blood-gas barrier, and pathogens are easy to pass through the barrier into the bloodstream. In addition, previous researches have revealed that urinary catheterization, admission to ICU, having a CVC, prior receipt of antibiotics, chemotherapy/radiation therapy, renal diseases are also independent risk factors for CRKP BSI. ${ }^{26,32-34}$ The discrepancies between our results and these findings might be due to different experimental designs, basic characteristics of the study population, sample sizes or statistical methods. In this study, we targeted the population with hematologic malignancies, so some factors might have no statistical significance and need to be further verified.

CRKP BSI results from a combination of factors, so the establishment of a relevant prediction model should be based on the screening of high-risk factors. This retrospective study analyzed more than 40 clinicopathological factors that might be associated with CRKP BSI. A risk prediction equation was built to judge the BSI risk rapidly in the clinical and implement the early intervention. We found that the prediction probability of CRKP BSI was $48 \%, 86 \%$ and $94 \%$ in patients with a total risk score of 5 , 7 and 8, respectively. It has important therapeutic implications if the risk score $\geq 5$. The score effectively stratified the risk of CRKP BSI, demonstrated by an AUROC of 0.753 $(95 \% \mathrm{CI}=0.648-0.858)$ which was higher than using CRKP rectal colonization alone $($ AUROC $=0.673 ; 95 \%$ $\mathrm{CI}=0.537-0.808)$.

Fosfomycin susceptibility testing was not routinely performed, only per request of the clinicians or in cases of extensive drug resistance. Márió Gajdács showed that some CRKP isolates were also susceptible to fosfomycin. ${ }^{35}$ It is possible to use fosfomycin as an alternative (if susceptibility is verified) to treat CRKP infections in combination therapy.

There were several limitations of this study that should be mentioned. Firstly, it was a single-center retrospective study; therefore, the results may not necessarily apply to other settings; there might be cases of CRKP BSI with negative blood culture results were not included for analysis. Secondly, the date when the first positive blood culture was drawn was considered as the onset of BSI, and it might be inconsistent with the real situation which may have happened earlier and thus affected the outcomes. Thirdly, although our prediction model has a good fit and predictive ability recognized separately by HosmerLemeshow test and ROC curve, our study did not perform an external validation of the prediction model which is one of our goals for further study.

\section{Conclusions}

CRKP BSI in patients with hematologic malignancies is associated with poor prognosis. The appropriate anti-infective 
treatment might play a crucial role in better survival. The risk prediction model in this study might help clinicians to detect the high-risk CRKP BSI patients accurately and administer effective CRKP-active therapy rapidly. As an important risk factor of CRKP BSI, CRKP rectal colonization should be actively screened routinely in patients with hematologic malignancies.

\section{Abbreviations}

IQI, 25-75\% interquartile interval; AML, acute myeloid leukemia; ALL, acute lymphoblastic leukemia; MDS, myelodysplastic syndrome; CLL/SLL, chronic lymphocytic leukemia/small lymphocytic lymphoma; CML, chronic myeloid leukemia; CMML, chronic myelomonocytic leukemia; NHL, non-Hodgkin's lymphoma; HSCT, hematopoietic stem-cell transplantation; GVHD, graft versus host disease; ANC, absolute neutrophil count; GI, gastrointestinal; CRKP, carbapenem-resistant Klebsiella pneumoniae; BSI, bloodstream infection; ICU, intensive care unit; IMV, invasive mechanical ventilation; PICC, peripherally inserted central catheter; CVC, central venous catheter; OR, odds ratio.

\section{Funding}

This work was supported by the research grant from the National Natural Science Foundations of China (No. NSFC81871689).

\section{Disclosure}

The authors declare that they have no competing interests for this work.

\section{References}

1. Gajdacs M, Albericio F. Antibiotic resistance: from the bench to patients. Antibiotics (Basel). 2019;8.

2. Gajdács M. The concept of an ideal antibiotic: implications for drug design. Molecules. 2019;24:892. doi:10.3390/molecules24050892

3. Hu F, Zhu D, Wang F, Wang M. Current status and trends of antibacterial resistance in China. Clin Infect Dis. 2018;67:S128-S34. doi:10.1093/cid/ciy657

4. Hu F, Guo Y, Yang Y, et al. Resistance reported from China antimicrobial surveillance network (CHINET) in 2018. Eur J Clin Microbiol Infect Dis. 2019;38(12):2275-2281. doi:10.1007/s10096-019-03673-1

5. Fasciana T, Gentile B, Aquilina M, et al. Co-existence of virulence factors and antibiotic resistance in new Klebsiella pneumoniae clones emerging in south of Italy. BMC Infect Dis. 2019;19:10. doi:10.1186/ s12879-019-4565-3

6. Satlin MJ, Jenkins SG, Walsh TJ. The global challenge of carbapenem-resistant Enterobacteriaceae in transplant recipients and patients with hematologic malignancies. Clin Infect Dis. 2014;58:1274-1283. doi:10.1093/cid/ciu052
7. Misch EA, Andes DR. Bacterial infections in the stem cell transplant recipient and hematologic malignancy patient. Infect Dis Clin North Am. 2019;33:399-+. doi:10.1016/j.idc.2019.02.011

8. Xu B, Huang SM, He LL, et al. Hematologic malignancies and carbapenem resistance: a multicentre retrospective study from Chinese people. Blood. 2017;130:1.

9. States U Antibiotic resistance threats in the United States, 2013; 2013

10. Trecarichi EM, Tumbarello M, Di Blasi R, et al. Bloodstream infections caused by Klebsiella pneumoniae in onco-hematological patients: incidence and clinical impact of carbapenem resistance in a multicentre prospective survey. Blood. 2015;126(23):3. doi:10.1182/blood.V126.23.3757.3757

11. Girmenia C, Rossolini GM, Piciocchi A, et al. Infections by carbapenem-resistant Klebsiella pneumoniae in SCT recipients: a nationwide retrospective survey from Italy. Bone Marrow Transplant. 2015;50:282-288. doi:10.1038/bmt.2014.231

12. Tofas P, Skiada A, Angelopoulou M, et al. Carbapenemase-producing Klebsiella pneumoniae bloodstream infections in neutropenic patients with haematological malignancies or aplastic anaemia: analysis of 50 cases. Int J Antimicrob Agents. 2016;47:335-339. doi:10.1016/j. ijantimicag.2016.01.011

13. Trecarichi EM, Pagano L, Martino B, et al. Bloodstream infections caused by Klebsiella pneumoniae in onco-hematological patients: clinical impact of carbapenem resistance in a multicentre prospective survey. Am J Hematol. 2016;91:1076-1081. doi:10.1002/ajh.24489

14. Webb BJ, Healy R, Majers J, et al. Prediction of bloodstream infection due to vancomycin-resistant enterococcus in patients undergoing leukemia induction or hematopoietic stem-cell transplantation. Clin Infect Dis. 2017;64:1753-1759. doi:10.1093/cid/cix232

15. Pagano L, Caira M, Trecarichi EM, et al. Carbapenemase-producing Klebsiella pneumoniae and hematologic malignancies. Emerg Infect Dis. 2014;20:1235-1236. doi:10.3201/eid2007.130094

16. Satlin MJ, Calfee DP, Chen L, et al. Emergence of carbapenem-resistant Enterobacteriaceae as causes of bloodstream infections in patients with hematologic malignancies. Leuk Lymphoma. 2013;54:799-806. doi:10.3109/10428194.2012.723210

17. Gajdacs M, Batori Z, Abrok M, Lazar A, Burian K. Characterization of resistance in gram-negative urinary isolates using existing and novel indicators of clinical relevance: a 10-year data analysis. Life (Basel). 2020;10:16.

18. Liu J, Wang H, Huang Z, et al. Risk factors and outcomes for carbapenem-resistant Klebsiella pneumoniae bacteremia in onco-hematological patients. J Infect Dev Ctries. 2019;13:357-364. doi:10.3855/jidc. 11189

19. Corcione S, Angilletta R, Raviolo S, et al. Epidemiology and risk factors for mortality in bloodstream infection by CP-Kp, ESBL-E, Candida and CDI: a single center retrospective study. Eur J Intern Med. 2018;48:44-49. doi:10.1016/j.ejim.2017.10.015

20. Satlin MJ, Jenkins SG, Chen L, et al. Septic shock caused by Klebsiella pneumoniae carbapenemase-producing Enterobacter gergoviae in a neutropenic patient with leukemia. J Clin Microbiol. 2013;51:2794-2796

21. Tumbarello M, Spanu T, Caira M, et al. Factors associated with mortality in bacteremic patients with hematologic malignancies. Diagn Microbiol Infect Dis. 2009;64:320-326. doi:10.1016/j. diagmicrobio.2009.02.008

22. Tang YS, Cheng Q, Yang Q, et al. Prognostic factors and scoring model of hematological malignancies patients with bloodstream infections. Infection. 2018;46:513-521. doi:10.1007/s15010-0181151-3

23. Tumbarello M, Viale P, Viscoli C, et al. Predictors of mortality in bloodstream infections caused by Klebsiella pneumoniae carbapenemase-producing K. pneumoniae: importance of combination therapy. Clin Infect Dis. 2012;55:943-950. doi:10.1093/cid/ cis 588 
24. Daikos GL, Tsaousi S, Tzouvelekis LS, et al. Carbapenemaseproducing Klebsiella pneumoniae bloodstream infections: lowering mortality by antibiotic combination schemes and the role of carbapenems. Antimicrob Agents Chemother. 2014;58:2322-2328. doi:10.1128/AAC.02166-13

25. Tumbarello M, Trecarichi EM, De Rosa FG, et al. Infections caused by KPC-producing Klebsiella pneumoniae: differences in therapy and mortality in a multicentre study. $J$ Antimicrob Chemother. 2015;70:2133-2143. doi:10.1093/jac/dkv086

26. Giannella M, Trecarichi EM, De Rosa FG, et al. Risk factors for carbapenem-resistant Klebsiella pneumoniae bloodstream infection among rectal carriers: a prospective observational multicentre study. Clin Microbiol Infect. 2014;20:1357-1362. doi:10.1111/1469-0691. 12747

27. Amit S, Mishali H, Kotlovsky T, Schwaber MJ, Carmeli Y. Bloodstream infections among carriers of carbapenem-resistant Klebsiella pneumoniae: etiology, incidence and predictors. Clin Microbiol Infect. 2015;21(1):30-34. doi:10.1016/j.cmi.2014.08. 001

28. Bellot P, Frances R, Such J. Pathological bacterial translocation in cirrhosis: pathophysiology, diagnosis and clinical implications. Liver Int. 2013;33:31-39. doi:10.1111/liv.12021

29. Wang F, Li Q, Wang C, Tang C, Li J. Dynamic alteration of the colonic microbiota in intestinal ischemia-reperfusion injury. PLoS One. 2012;7:e42027. doi:10.1371/journal.pone.0042027
30. Kollef MH, Sherman G, Ward S, Fraser VJ. Inadequate antimicrobial treatment of infections - a risk factor for hospital mortality among critically ill patients. Chest. 1999;115(2):462-474. doi:10.1378/ chest.115.2.462

31. Cattaneo C, Di Blasi R, Skert C, et al. Bloodstream infections in haematological cancer patients colonized by multidrug-resistant bacteria. Ann Hematol. 2018;97(9):1717-1726. doi:10.1007/s00277018-3341-6

32. Borer A, Saidel-Odes L, Eskira S, et al. Risk factors for developing clinical infection with carbapenem-resistant Klebsiella pneumoniae in hospital patients initially only colonized with carbapenem-resistant K pneumoniae. Am J Infect Control. 2012;40:421-425. doi:10.1016/j. ajic.2011.05.022

33. Gasink LB, Edelstein PH, Lautenbach E, Synnestvedt M, Fishman NO. Risk factors and clinical impact of Klebsiella pneumoniae carbapenemase-producing K. pneumoniae. Infect Control Hosp Epidemiol. 2009;30:1180-1185. doi:10.1086/648451

34. Orsi GB, Garcia-Fernandez A, Giordano A, et al. Risk factors and clinical significance of ertapenem-resistant Klebsiella pneumoniae in hospitalised patients. J Hosp Infect. 2011;78:54-58. doi:10.1016/j. jhin.2011.01.014

35. Gajdacs M, Abrok M, Lazar A, Burian K. Comparative epidemiology and resistance trends of common urinary pathogens in a tertiary-care hospital: a 10-year surveillance study. Medicina (Kaunas). 2019;55:356.
Infection and Drug Resistance

\section{Publish your work in this journal}

Infection and Drug Resistance is an international, peer-reviewed openaccess journal that focuses on the optimal treatment of infection (bacterial, fungal and viral) and the development and institution of preventive strategies to minimize the development and spread of resistance. The journal is specifically concerned with the epidemiology of antibiotic resistance and the mechanisms of resistance development and diffusion in both hospitals and the community. The manuscript management system is completely online and includes a very quick and fair peerreview system, which is all easy to use. Visit http://www.dovepress.com/ testimonials.php to read real quotes from published authors. 